\title{
Analysis of Sleep-Mode Downlink Scheduling Operations in EPON Systems
}

\author{
Yan, Ying; Dittmann, Lars
}

Published in:

Proceedings of The Eighth International Conference on Information, Communications, and Signal Processing (ICICS 2011)

Publication date:

2011

Link back to DTU Orbit

Citation (APA):

Yan, Y., \& Dittmann, L. (2011). Analysis of Sleep-Mode Downlink Scheduling Operations in EPON Systems. In Proceedings of The Eighth International Conference on Information, Communications, and Signal Processing (ICICS 2011) http://www.icics.org/2011/

\section{General rights}

Copyright and moral rights for the publications made accessible in the public portal are retained by the authors and/or other copyright owners and it is a condition of accessing publications that users recognise and abide by the legal requirements associated with these rights.

- Users may download and print one copy of any publication from the public portal for the purpose of private study or research.

- You may not further distribute the material or use it for any profit-making activity or commercial gain

- You may freely distribute the URL identifying the publication in the public portal

If you believe that this document breaches copyright please contact us providing details, and we will remove access to the work immediately and investigate your claim. 


\title{
Analysis of Sleep-Mode Downlink Scheduling Operations in EPON Systems
}

\author{
Ying Yan and Lars Dittmann \\ Department of Photonics \\ Technical University of Denmark, Denmark \\ Email: \{yiya,ladit\}@fotonik.dtu.dk
}

\begin{abstract}
Energy management strategy has been considered as an important component in the future Ethernet Passive Optical Networks (EPONs). In this paper, a sleeping mode operation is studied, and a downlink packet scheduling scheme is analyzed to preserve energy consumption and maintain the required QoS. This paper proposes a novel sleep-mode downlink packet scheduling scheme in order to enhance the sleep control function. Simulation results confirm that the proposed approach offers effective power management on the basis of the traffic conditions. The trade-off between network performances and the power saving is examined and compared.
\end{abstract}

Keywords-EPON; Energy Efficiency; Sleep-Mode;

\section{INTRODUCTION}

EPONs have received a worldwide deployment to provide FTTx (x stands for home, building, curb, etc.) because of the bandwidth enhancement and low maintenance cost offered by optical fibers. Energy efficiency is one of the main design aspects in the future EPON system. Recent research has shown that broadband access networks consume a high share of the total energy consumption by all telecommunication equipments [1-2]. Although EPON consumes less power compared to other wired access technologies, it has raised attentions to further preserving the power consumption, especially when the line rate is increased and the number of users is continuously grown.

A typical EPON system consists of one Optical Line Terminal (OLT) functionalized as a central office, one passive optical splitter implemented in the remote node, and multiple Optical Network Units (ONUs) residing at subscribers' locations. In the shared medium optical network, each ONU transmits within the assigned time slots in the upstream direction. In the downstream direction, an ONU filters and selectively accepts the broadcast packets. As shown in Figure 1 , the major sources of energy waste include the overhearing (which means that an ONU receives and decodes packets that are not destined to it) and the idle listening (which means an ONU waiting for the control message). It is obvious that the ONUs consume useless energy due to the lack of an energy management mechanism in the original EPON.

A simple method for power saving is to transit the ONU into a sleep mode when there is neither upstream nor downstream transmission. During a sleep period, ONUs turn off the transceiver in order to save energy. However, some functions, such as clock and data recovery and back-end digital circuit, are still powered on. A timer is utilized to count down the sleeping time in the power saving control. Upon receiving

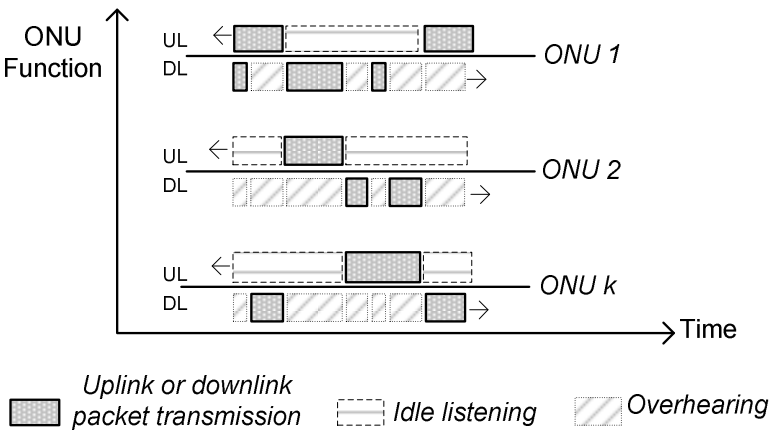

Figure 1. ONU operations in the EPON system

notification of entering the sleep mode, the timer is triggered and the ONU wakes up when the timer is expired.

After entering the sleep mode, ONUs are in the absence of traffic at the optical transmission interface. In case there is incoming traffic, packets are buffered and prescheduled for upstream and downstream transmission in both the OLT and ONUs. ONUs need to wake up and exchange traffic with the OLT. However, a long sleep period may result in the QoS degradation, especially for the high priority services. On the other hand, a short sleeping period is helpful to improve the delay, but it results in a problem of frequent transition between the sleep and active mode. Hence, the sleep period should be carefully scheduled in order to avoid missing any incoming packets and complete transmission without violating QoS requirements.

Several studies have been recently proposed to analyze the power consumption for EPON, while applying the approach of allowing ONUs to sleep mode [3-5]. In this paper, a sleepmode downlink packet scheduler is proposed. The basic idea is that the OLT schedules the queued packet in the downstream transmission on the basis of the traffic conditions and the sleep mode of the destined ONU. The proposed mechanism applies both the weighted queuing technique and a joint sleep-mode scheduling to achieve effective power management and reduced switchover overhead. Additionally the sleep period is assigned with a minimum value for the lightly loaded ONUs, so that the total power saving is improved. We focus on the protocol design of the energy management mechanism and the scheduling strategy to maximize energy saving.

The rest of the paper is organized as follows. In Section II, we present the system model and the sleeping period scheduling algorithms. Section III presents a novel sleep-mode 
downlink scheduling scheme. An enhanced sleeping window decision scheme is presented in Section IV; this scheme improves the energy efficiency when the ONU is under a low volume of incoming traffic. Section V presents the performance evolution, and finally, we conclude in Section VI.

\section{SLEEP TIME SCHEDULING SCHEMES IN EPON}

\section{A. System Model}

In this paper, we consider an EPON system consisting of an OLT, $1: K$ splitter and multiple ONUs. TDMA is used in the physical layer where bandwidth is divided in time slots. Each ONU maintains an upstream buffer and sends upstream data to the OLT during assigned time slots. In the downstream direction, data are queued in the OLT and transmitted when the downstream bandwidth is available. The power consumption in the active state includes energy consumed in listening to the OLT and in receiving or transmitting data. Assuming that the overall transmission period is divided into $N$ time slots, ONUs are either in active mode or in sleep mode during the time slot $n$. The total energy expenditure model is formalized as follows:

$$
\text { Energy }=\sum_{i=1}^{M} \sum_{n=1}^{N} T_{i}^{n} \cdot\left[s_{i}^{n} \cdot P_{\text {active }}+\left(s_{i}^{n}-1\right) P_{\text {sleep }}\right](1)
$$

Within a period, the total energy consumption includes energy consumed by all ONUs in both the active $\left(P_{\text {active }}\right)$ and sleep state $\left(P_{\text {sleep }}\right)$. The $T^{n}{ }_{i}$ represents the period of time slot $n$ in ONU $i$ and $s_{i}^{n}$ represents the state indication in time slot $n$.

Following our model, it is obvious that energy depletion is determined by the time the ONU spend in the active mode. The objective of this work is to extend the sum of the sleep period and meanwhile provide the transmission with QoS guarantees.

\section{B. $M P C P$ in $E P O N$}

The IEEE 802.3ah Task Force [6] has developed and standardized a signaling access protocol, MultiPoint Control Protocol (MPCP) for the EPON system. The OLT gathers information from the associated ONUs and allocates bandwidth resources to ONUs using the polling and granting mechanism. The time interval between the two successive polling activities in one ONU is defined as a Cycle $\left(T_{\text {cycle }}\right)$ in this paper. Within each cycle, ONUs report its bandwidth requirements according to its buffer occupancy to the OLT via a REPORT message. The OLT updates the status of ONUs based on the received REPORT message, and performs the appropriate bandwidth allocation algorithm to assign transmission windows to ONUs. The transmission grants are broadcast via a GATE message, containing the transmission information such as the start time $\left(T_{\text {start }}\right)$ and the end time $\left(T_{\text {end }}\right)$.

The signaling mechanism between the OLT and ONUs is defined in the standardized MPCP, but the bandwidth allocation algorithm is not specified but open for alternative implementations. Various bandwidth allocation schemes have been proposed and designed using fixed allocation scheme or Dynamic Bandwidth Allocation (DBA) scheme [7]. The DBA assign the upstream bandwidth for different ONUs according to its bandwidth request. The bandwidth reservation technique improves the resource utilization and guarantees the bandwidth demand.

\section{MPCP based Sleep Time Scheduling (STS) Scheme}

We briefly describe the MPCP based Sleep Time Scheduling (STS) scheme here. Details are discussed in our previous paper [8]. In the next section, improvements are proposed by using a sleep-mode downlink packet scheduler.

Our proposed energy management mechanism is based on the services offered by the MPCP. A STS scheme is deployed in the OLT to assign the sleep time and wakeup time to each ONU with considerations of both upstream and downstream transmission. The OLT gathers information from the ONUs, allocates uplink/downlink bandwidth to ONUs, and performs the sleep period assignment. The framework of the STS mechanism is depicted in Figure 2.

The OLT maintains an entry table for both the bandwidth allocation and the sleep time scheduling (as illustrated as Figure 2a). The original GATE message is modified as the sleep-mode GATE message, Gs, which carries additional parameters: the start time for the sleep mode ( $\left.t_{\text {sleep }}\right)$ and the wakeup time ( $\left.t_{\text {wakeup }}\right)$. The downstream packets are queued in a First-In First-Out fashion in the OLT (as shown in Figure 2b).

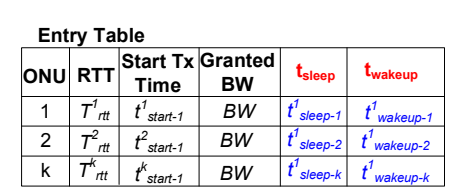

(a)

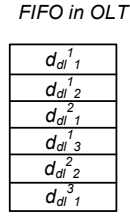

(b)

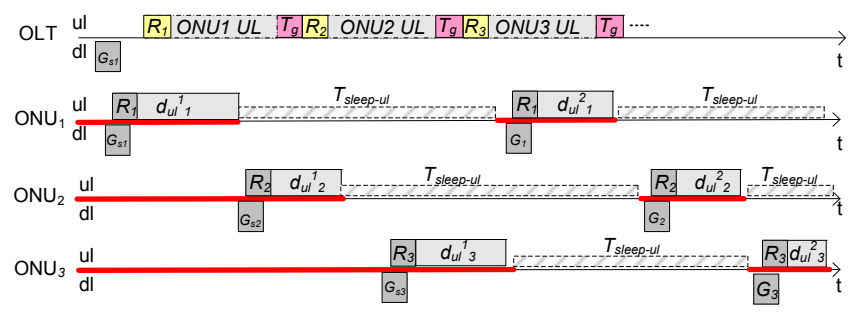

(c) assign sleep period based on upstream bandwidth allocation

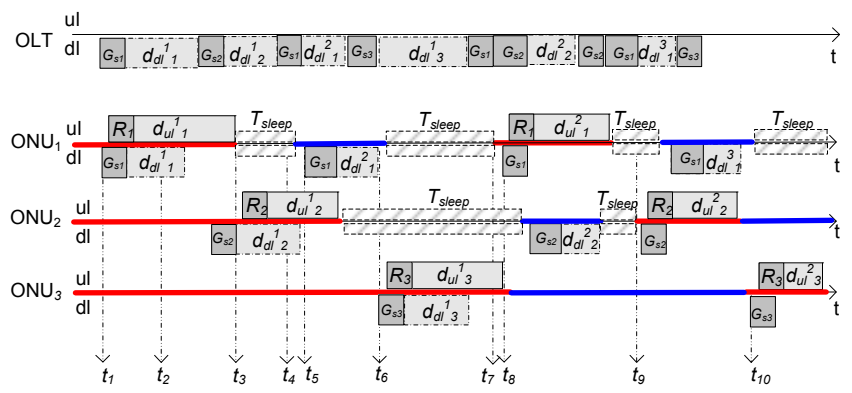

(d) assign sleep period based on downstream bandwidth allocation G GATE R REPORT d $\begin{aligned} & d_{u l i}{ }_{i}, d_{d l}{ }_{i} \text { : Uplink or Downlink data } \\ & \text { for the ith ONU in the nth cycle }\end{aligned}$

Figure 2. MPCP based Sleep Time Scheduling (STS) scheme 
The sleep time scheduling scheme has two steps operations. Firstly, as shown in Figure 2c, the sleep period is determined based on the upstream transmission $\left(T_{\text {sleep-ul }}\right)$. The OLT grants the upstream bandwidth by polling each ONU. During the granted upstream transmission period, the ONU should be awake (the active period is marked as red color). Secondly, as shown in Figure 2d, the downstream traffic is taken into account. The wakeup time is precisely calculated and assigned, so that the ONU can switch to the active mode and carry out the next upstream or downstream transmission. The sleep period is reduced because of the active period for the downstream transmission (the additional active period for the downstream traffic is marked as blue color). Since the operations of the above scheduling mechanism requires the OLT to locate the next packet in the buffer in order to calculate the wakeup time, ONUs which have no more queued packets cannot enter the sleep mode. They should keep awake to listen to the notification and cannot enter to sleep until any explicit assignment of the wake up time is received (e.g. $\mathrm{ONU}_{3}$ need to keep the active state from $t_{8}$ to $t_{10}$ in Figure $2 \mathrm{~d}$ ).

\section{ENHANCECD DOWNLINK PACKET SCHEDULING SCHEME}

\section{A. Problem Statement}

One problem is to minimize the switchover frequency, because the switchover process takes extra time and consumes power. As observed from our previous design, the sleep time scheduling scheme calculates the sleep and the wake up time without consideration of the switchover penalty. For example, as shown in Figure $2 \mathrm{~d}$, the $O N U_{1}$ receives data $d_{d l}{ }_{1}$ during its active period. Then the downlink packet scheduler grants transmission to the data $\left(d_{d l}^{l} 2\right)$ for the ONU2. Following the FIFO fashion, the buffered data $\left(d_{d l}{ }^{2}\right)$ is sent afterwards, which results in a state switchover in the $O N U_{l}$.

In order to overcome this problem, downstream packets should be differentiated based on the sleep status of their destined ONU. For the downstream traffic, FIFO is simple to implement but lack of an intelligent control. In order to reduce the frequency of state switchover, advanced queuing technique and packet scheduling scheme on the downstream channel need to be taken into account in the sleep time assignment. Additionally, the delay sensitive applications (real-time traffic) should be treated separately in order to satisfy their restrict QoS requirements.

\section{B. Scheduler Design}

In this paper, a sleep-mode scheduling algorithm for downlink traffic is proposed, namely Sleep-mode Downlink Packet Scheduling (SDPS), which is a modified scheduling algorithm based on a weight factor. This algorithm is applied directly to the queued data in the OLT, which calculates the transmission time of each packet and the QoS. Moreover, the proposed algorithm works along with the sleep time scheduler to detect the sleep mode of each ONU and reduce the potential switchover frequency.

SDPS uses a "weight" parameter, $\alpha_{i}$, which defines the selection probability of to the queue. The scheduling decision of the output traffic is based on the value of $\alpha_{i}$. A Round Robin scheduler visits each nonempty queue and calculates the request bandwidth and the QoS requirements (delay and the minimum throughput). Three types of weight factor are assigned based on conditions as following.

- Condition 1: The newly arriving downlink packets are classified and buffered into the corresponding priority queues based on the Class of Service $(\mathrm{CoS})$ identifier. The high priority queue obtains a high weight factor, $\alpha \_c o s \_i$.

- Condition2: The OLT keeps a track of the sleep status of each ONU. The packets that destined to an active ONU obtain a highweight factor, $\alpha \_$active_ $i$.

- Condition3: There is a basic weight factor, $\alpha$ queue_ $i$, associated with each incoming packet, which increases along with the queuing time.

The advantage of the SDPS scheme in particular is that the OLT can adaptively set the weight factors depending on the experienced traffic and the service class type in different phases, such as the sleep state or the awake state. Hence, for the delay sensitive traffic, packets are granted with the downlink bandwidth with a high priority. For the low priority traffic, the SDPS proportionally distributes bandwidth among ONUs in the active state and sleep state. Using the weight factor, the packet destined to an active ONU can be transmitted during the awake period, although other packets destined to a sleep ONU have been queued for a longer period.

However, the calculation for the sleep period of an ONU becomes more complex in the downstream direction. At the scheduling instant, the OLT allocates bandwidth to packets according to their weight factors. The next wake up time is calculated based on the queue occupancy destined to other ONUs and the sleep status. At time $t$, the OLT transmits a packet to the $O N U_{i}$. If there are more packets queued for the $O N U_{i}$, the OLT first examines the remained request bandwidth in the high priority queues. Then the OLT checks the weight factor of the next queued data and compared it with the weight factors of data in other queues. Therefore the transmission time for the next queued data can be estimated and informed to the $O N U_{i}$ as the next wake up time.

If the number of remaining queued data is none, the next wake up time will be assigned as none. Note that when the $O N U_{i}$ wakes up at the predetermined time, the high priority packet will be transmitted as long as the high priority queue is not empty. The description of the proposed algorithm is listed in ALGORITHM 1.

\section{Minimum Sleep Window for the Lightly-loaded ONU}

As mentioned in Section II, the sleep and wakeup time must be specified and assigned before the ONU enters the sleep state, because the ONU turns off the transceiver and is unable to sense the channel. Without knowledge of the exact downlink traffic information, the OLT cannot estimate the next wake up time. As a result, the system QoS has to be sacrificed if the ONU stays in the sleep mode for long. On the other hand, the energy saving is reduced if the ONU remains in the active mode and wait for the notification. As such, we slightly modify the sleep window assignment as follows. 


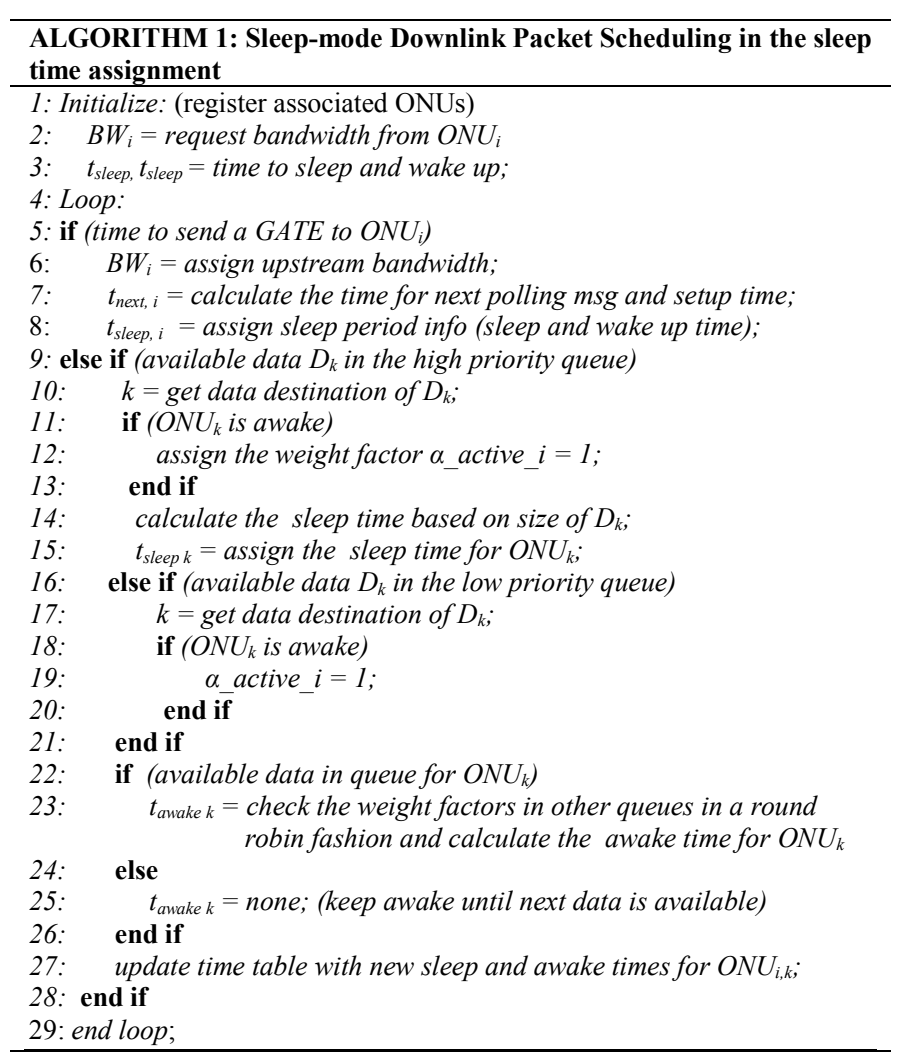

When an ONU is lightly loaded, which in turn means a small amount of downstream incoming traffic, the OLT cannot always determine the wakeup time for next downstream packets based on the buffered data. Under this circumstance, the OLT assign the ONU a minimum value of sleep window $\left(T_{\text {min }}\right)$ rather than keep the ONU awake. As shown in Fig.3, the sleep period is increased if the ONU continues sleep mode (e.g. $T_{\text {min }}, 2 T_{\text {min }}$ ), until it starts the next transmission (at $t_{\text {poll } 2}$ ). After the first sleep interval, the ONU transits into awake statues and listens to the traffic indication from the OLT. If there is no traffic, the ONU continues sleep mode, the next sleep window is double the preceding sleep interval. That is the duration of sleep interval in the nth sleep period is given $T_{n}$ $=2^{n-1} T_{\min }$.

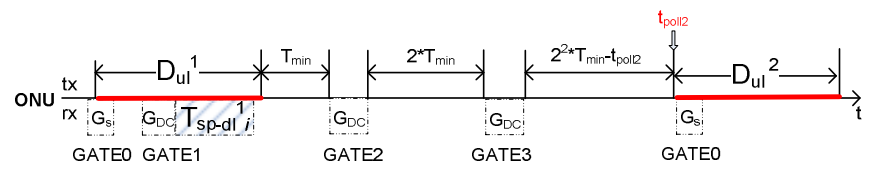

Figure 3. Schematic of the sleep mode with minimum length

\section{SimUlation RESUltS}

This section describes the simulations conducted to confirm the improvements on the network performances of the sleepmode downlink packet scheduling scheme. The simulation is performed using the OPNET modeler [9].

\section{A. Simulation Model}

We have assumed that the OLT is connected to 32 ONUs, which are enabled with power saving functionality. The optical link rate is 1 Gbps for both upstream and downstream transmission. The guard time between two consecutive transmission slots is 5 us. For allocating upstream bandwidth among ONUs, both the static schemes are implemented at the OLT because the downstream transmission is the focus of this test. The basic transmission cycle is set to $2 \mathrm{~ms}$. Traffic is generated following Poisson distribution and the length of packets is generated independently between 64 bytes to 1200 bytes. Destinations of downstream traffic are uniformly distributed among connected ONUs. The downstream traffic load is varied in order to test our proposed downlink packet scheduling algorithm. The MPCP control messages, GATE and REPORT, are formatted according to the IEEE 802.3ah specification.

We study performances and features of the power saving mechanism, STS, with and without the proposed SDPS compared to the Power Ignoring Scheme (PIS) and the Upstream Centric Scheduling (UCS) scheme. The UCS scheme schedules the sleep period based on the upstream bandwidth allocation scheme, and the downstream traffic can be transmitted only when the destined ONU is active [8].

\section{B. Performance Analysis}

The goal of the power saving mechanism is to reduce power consumption and maintain the network performance. For this purpose, we assess the system performance according to the power consumption, the average queuing delay, and the switchover frequency. The average awake time in ONUs is used as a merit for energy efficiency performances. The lower the ratio of the awake time to the total observation period represents the better energy efficiency.

The results in Figure 4 show the power consumption. In the case of PIS, ONUs constantly stay in awake state and consumes high energy (100\% of the observation period). Particularly, using the UCS scheme saves up to $90 \%$ power on time. The energy consumption is increased in the STS case because of the additional active period for the downstream traffic. With the assistance of our proposed SDPS scheme for the downstream traffic, the power consumption is reduced. Owing to the weight factor, the queued data with an active destination is transmitted with high preferences. However, when the traffic load is increased, the number of the transmission data to the active ONU is reduced because other packets should be transmitted at first in order to avoid QoS violation.

The QoS performance in terms of the average queuing delay in the OLT is shown in Figure 5. The average delay of the STS scheme is better than that of the UCS scheme since the downstream packets have to wait for their awake period in the OLT buffer in the UCS scheme. With 32 ONUs in the EPON system, the cycle period causes a large amount of queuing delay. As observed, with the SDPS assistance, the real-time traffic is transmitted with a high priority and experiences very low delay even when the traffic load becomes high. 


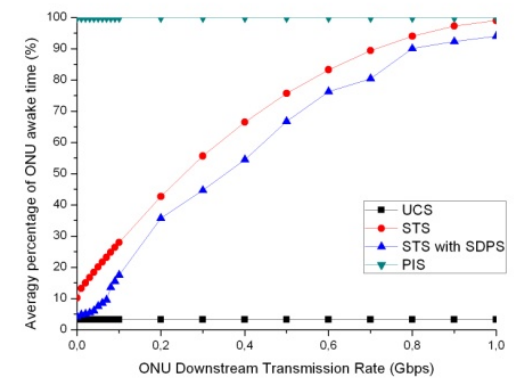

Figure 4. Simulation results - power consumption

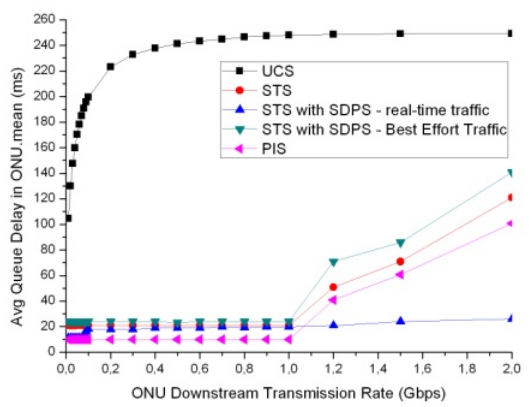

Figure 5. Simulation results - average queuing delay in OLT

The switchover frequency is used as a metric in the performance analysis. The results of the switchover process are highly depended on the traffic pattern. The number of switchover is recorded in Figure 6. The performance is compared by varying the allocated upstream bandwidth. When the assigned upstream awake period is increased, there are more data can be send during the active period. However, when the upstream frame becomes large, it increases the switchover frequency because the cycle period becomes large. The ONU has to wake up more times during its sleep period and receive the downlink packets, which in turn increases the switchover frequency. Actually the reduce of the switchover frequency can also be confirmed from the energy consumption result (shown in Figure 4). When the switchover frequency becomes less, the number of the switchover overhead is reduced. Thus the power saving is improved.

At last we test a hybrid mechanism including the SDPS and the minimum sleep period for the lightly loaded ONUs. There are 16 ONUs with lightly loaded downlink traffic. As shown in Figure 7, all ONUs are awake in the PIS case and ONUs are awake in turn in the UCS case. However, the energy consumption is high, when the STS with and without the advanced downlink packet scheduler is used, because the lightly loaded ONUs keep in the active state as long as there is no queued downstream packet. By deploying the minimum sleep window, the energy efficiency is greatly improved.

\section{CONCLUSION}

In this paper, we discuss a downlink packet scheduling problem focusing on minimizing energy consumption of ONUs and providing QoS guarantee for the real-time traffic. In order

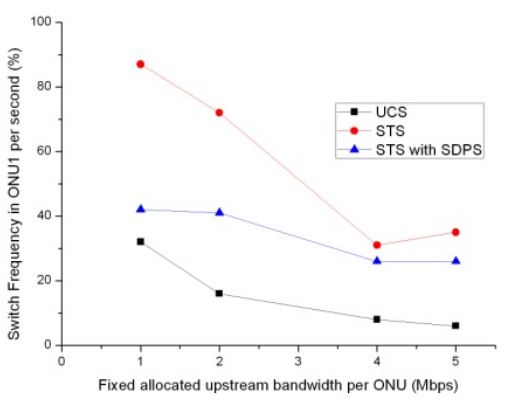

Figure 6. Simulation results - Swithover Frequency

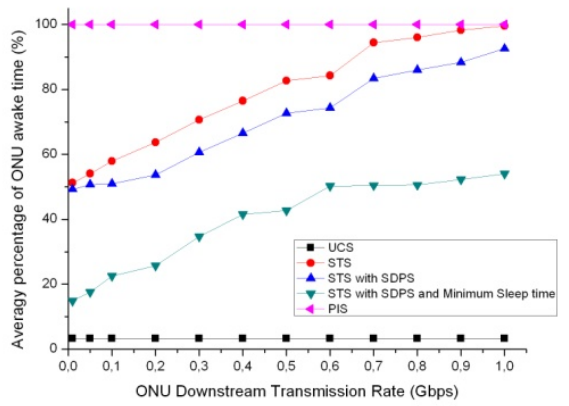

Figure 7. Simulation results - power consumption in the lightly load scenario

to clearly formulate the relationship between the sleep time scheduling and the traffic condition, we use the weighted scheduling formulation. Through simulation results, it was confirmed that the proposed sleep-mode scheduler can increase the energy efficiency and improve the network performance.

[1] T. Smith, R. S. Tucker, K. Hinton, and A. V. Tran, "Implications of sleep mode on activation and ranging protocols in PONs," 21st Annual Meeting of the IEEE Lasers and Electro-Optics Society (LEOS), vol. 11, pp. 604-605, 2008

[2] M. Gupta and S. Singh, "Greening of the internet," in Proc. ACM SIGCOMM '03, pp.19-26, Aug. 2003

[3] J. Mandin, "EPON Powersaving via Sleep Mode," IEEE 802.3az Meeting, September 2008.

[4] R. Kubo, J. Kani, Y. Fujimoto, N. Yoshimoto, and K. Kumozaki, "Proposal and Performance Analysis of a Power-Saving Mechanism for 10 Gigabit Class Passive Optical Network Systems," in NOC, June 2009.

[5] S. Wong, L. Valcarenghi, S. Yen, D. Campelo, S. Yamashita, and L. Kazovsky, "Sleep Mode for Energy Saving PONs: Advantages and Drawbacks," Globlecom Second International workshop on Green Communication, November 2009.

[6] IEEE 802.3ah, Ethernet in the First Mile Task Force. [online] http://www.ieee802.org/3/efm/index.html

[7] Kramer Glen, Mukherjee Biswanath, and Pesavento Gerry. "IPACT: a dynamic protocol for an Ethernet PON (EPON)," IEEE Communication Magazine 40(2), 74-80 (2002).

[8] Ying Yan and Lars Dittmann, "Energy Efficiency in Ethernet Passive Optical Networks (EPONs): Protocol Design and Performance Evaluation", Journal of Communications, Volume 6, Issue 3, pp. 249261, 2011.

[9] OPNET Modeler 14.5, http://www.opnet.com/, 2005 